\title{
86. DATA REPORT: URANIUM, THORIUM, AND OTHER TRACE ELEMENTS IN STRIP SAMPLES FROM CORES 128-798B-13H THROUGH -15H ${ }^{1}$
}

\author{
Caroline M. Isaacs ${ }^{2}$
}

\section{INTRODUCTION}

Because of heterogeneity, average values of sediment composition can be difficult to establish accurately in fine-grained rocks. As a result, derived values (such as biogenic silica accumulation rates) can be unreliable, and stratigraphic or spatial trends in composition indeterminate. Samples that reflect interval averages, such as well cuttings, have proven to be more valuable than conventional samples for determining compositional averages and trends reliably (Isaacs, 1987; Isaacs et al., 1990). If properly calibrated, geochemical and gamma-ray logs can also be useful.

As part of calibrating the gamma-ray and geochemical logs taken during Leg 128 (Ingle, Suyehiro, von Breymann, et al., 1990), a sampling method similar to that employed by LeClaire and Kelts (1982) was used. Channel or strip samples representing interval averages were obtained from Cores $128-798 \mathrm{~B}-13 \mathrm{H}$ through $-15 \mathrm{H}$, and analyzed for major oxides (Bristow and deMenocal, this volume).

Splits of these samples were also analyzed for $\mathrm{U}$ and Th to compare with data from the natural gamma-ray spectroscopy tool (GST, trademark of Schlumberger Inc.). At the same time, the splits were analyzed for a variety of other major and minor elements. These data are presented here (Table 1).

\section{METHOD}

Because of the small sample size available $(0.5-1.0 \mathrm{~g})$, the method used to analyze the samples was instrumental neutron activation analysis (INAA). All analyses were performed by X-ray Assay Laboratories in Don Mills, Ontario, Canada.

Samples were irradiated in batches of up to 60 samples in a neutron flux of about $4 \times 10^{12} \mathrm{n} / \mathrm{cm}^{2} / \mathrm{s}$, together with international standard reference materials (NIST, CANMET, and USGS) and a neutron flux monitoring foil. Each batch was wrapped in polyethylene and placed inside a watertight irradiation facility that was rotated continuously during irradiation. After a decay period of about seven days, the samples were counted for up to $2000 \mathrm{~s}$ on a high-purity germanium (HPGE) detector. Each detector has an efficiency of $>10 \%$ relative to an $\mathrm{NaI}$ detector, and an energy resolution exceeding $1.9 \mathrm{keV}$ (at $1332.5 \mathrm{keV}$ ). About $30 \%$ of the samples in each batch were recounted one to three days later for quality control. The flux monitoring foil was also counted on the HPGE detector, and the information used to compensate for any variation of the neutron flux during the irradiation.

The gamma-ray energy spectrum was transferred to a computer system for analysis. Peak identification, spectral interference corrections, and fission product corrections were determined using a customized version of trace element analysis by automated gamma

\footnotetext{
' Tamaki, K., Suyehiro, K., Allan, J., McWilliams, M., et al., 1992. Proc. ODP, Sci. Results, 127/128, Pt. 2: College Station, TX (Ocean Drilling Program).

${ }^{2}$ U.S. Geological Survey, 345 Middlefield Road, Ms 999, Menlo Park, CA 94025.
}

spectroscopy (TEABAGS). The elemental concentration was calculated from a weighted average of multiple peak data for each element.

Approximately $10 \%$ of the samples were run in duplicate. Specific data on duplicates for the samples reported here are not available. Average relative standard deviations for duplicates are presented in Table 2 for certain elements within the abundance ranges reported. These averages range from $0.0 \%$ for Cs to $12.9 \%$ for Mo.

To evaluate accuracy of the data, a separate set of 22 samples was analyzed using both INAA and inductively coupled atomic plasma spectroscopy (ICP) at X-ray Assay Laboratories, with agreement between the two techniques for common elements $(\mathrm{Fe}, \mathrm{Ca}, \mathrm{Na}, \mathrm{Co}$, $\mathrm{Cr}, \mathrm{Mo}, \mathrm{Sc}$, and $\mathrm{Zn}$ ) better than $\pm 3 \%$ at one standard deviation. The identical powders were also analyzed at X-ray Assay Laboratories by X-ray fluorescence spectroscopy (XRF) and at U.S. Geological Survey laboratories twice by XRF and once by ICP. Except for $\mathrm{Ba}$, this interlaboratory comparison showed excellent agreement. Data for these 22 samples (D.Z. Piper and C.M. Isaacs, unpubl. data) will be reported and evaluated elsewhere.

Method detection limits for each element reported here are shown in Table 2. Certain elements were below the detection limit in all samples ( $<200 \mathrm{ppm} \mathrm{Ni},<500 \mathrm{ppm} \mathrm{Sr},<20 \mathrm{ppb}$ Ir).

\section{ACKNOWLEDGMENTS}

This research was funded by the U.S. Science Support Program of the Joint Oceanographic Institutions, Inc. I especially thank Jim Ingle for his many determined efforts on behalf of the research. I also thank Peter deMenocal and Jim Bristow for collecting and splitting the samples.

\section{REFERENCES}

Ingle, J. C., Jr., Suyehiro, K., von Breymann, M. T., et al., 1990. Proc. ODP, Init. Repts., 128: College Station, TX (Ocean Drilling Program).

Isaacs, C. M., 1987. Use of well cuttings for determining compositional trends in the Monterey Formation, California. AAPG Bull., 71:570-571.

Isaacs, C. M., Tomson, J. H., Stewart, K. C., and Jackson, L. L., 1990. Abundances of major elements and sedimentary components of cuttings from the Foxen Mudstone and the Sisquoc, Monterey, and Point Sal Formations in the Union Hobbs 22 well, and preliminary comparison with cores from the Union Newlove 51 well, Orcutt oil field, onshore Santa Maria basin, California. Open-File Rept.-U.S. Geol. Surv., 89-466.

LeClaire, J. P., and Kelts, K. R., 1982, Calcium carbonate and organic carbon stratigraphy of late Quaternary laminated and homogeneous diatom oozes from the Guaymas Slope, HPC Site 480, Gulf of California. In Curray, J. R., Moore, D. G., et al., Init. Repts. DSDP, 64: Washington (U.S. Govt. Printing Office), 1263-1275. 
Table 1. Geochemical data by INAA on strip samples, Site 798B.

\begin{tabular}{|c|c|c|c|c|c|c|c|c|c|c|c|c|c|c|c|c|}
\hline $\begin{array}{l}\text { Core, } \\
\text { section }\end{array}$ & $\begin{array}{l}\text { Interval } \\
(\mathrm{cm})\end{array}$ & $\begin{array}{l}\text { ODP } \\
\text { depth } \\
\text { (mbsf) }\end{array}$ & $\begin{array}{c}\text { Corrected } \\
\text { depth } \\
\text { (mbsf) }\end{array}$ & $\underset{(\mathrm{ppm})}{\mathrm{Ag}}$ & $\begin{array}{c}\text { As } \\
\text { (ppm) }\end{array}$ & $\underset{(\mathrm{ppb})}{\mathrm{Au}}$ & $\underset{(\mathrm{ppm})}{\mathrm{Ba}}$ & $\begin{array}{c}\mathrm{Br} \\
(\mathrm{ppm})\end{array}$ & $\begin{array}{l}\mathrm{Ca} \\
(\%)\end{array}$ & $\underset{(\mathrm{ppm})}{\mathrm{Ce}}$ & $\begin{array}{c}\text { Co } \\
\text { (ppm) }\end{array}$ & $\underset{(\mathrm{ppm})}{\mathrm{Cr}}$ & $\underset{(\mathrm{ppm})}{\mathrm{Cs}}$ & $\begin{array}{c}\mathrm{Eu} \\
(\mathrm{ppm})\end{array}$ & $\begin{array}{l}\mathrm{Fe} \\
(\%)\end{array}$ & $\begin{array}{c}\mathrm{Hf} \\
(\mathrm{ppm})\end{array}$ \\
\hline $13 \mathrm{H}-1$ & $0-30$ & 113.50 & 113.65 & $<5$ & 21 & 11 & 1000 & 86 & 1.6 & 77 & 23 & 98 & 12 & 1.5 & 4.58 & 4.9 \\
\hline $13 \mathrm{H}-1$ & $30-60$ & 113.80 & 113.91 & $<5$ & 13 & 9 & 800 & 90 & 3.1 & 75 & 22 & 90 & 11 & 1.0 & 3.78 & 3.6 \\
\hline $13 \mathrm{H}-1$ & $60-90$ & 114.10 & 114.16 & $<5$ & 11 & 7 & 900 & 93 & 4.1 & 63 & 20 & 85 & 9 & 0.9 & 3.31 & 3.4 \\
\hline $13 \mathrm{H}-1$ & $90-119$ & 114.40 & 114.41 & $<5$ & 13 & $<5$ & 800 & 60 & 1.5 & 70 & 18 & 85 & 11 & 1.1 & 3.51 & 2.6 \\
\hline $13 \mathrm{H}-2$ & $0-25$ & 114.69 & 114.66 & $<5$ & 15 & $<5$ & 800 & 67 & 2.4 & 72 & 16 & 81 & 12 & 1.0 & 3.46 & 4.0 \\
\hline $13 \mathrm{H}-2$ & $32-61$ & 115.01 & 114.93 & $<5$ & 15 & $<5$ & 900 & 91 & 0.9 & 62 & 16 & 73 & 9 & 1.4 & 3.17 & 3.3 \\
\hline $13 \mathrm{H}-2$ & $61-100$ & 115.30 & 115.17 & $<5$ & 12 & 6 & 1100 & 78 & 1.3 & 60 & 15 & 77 & 10 & 1.0 & 3.04 & 3.4 \\
\hline $13 \mathrm{H}-2$ & $100-125$ & 115.69 & 115.50 & $<5$ & 13 & $<5$ & 800 & 72 & 1.3 & 63 & 17 & 76 & 11 & 0.9 & 3.25 & 4.0 \\
\hline $13 \mathrm{H}-2$ & $125-150$ & 115.94 & 115.72 & $<5$ & 11 & $<5$ & 700 & 81 & 1.3 & 64 & 17 & 72 & 10 & 0.7 & 3.08 & 3.4 \\
\hline $13 \mathrm{H}-3$ & $0-30$ & 116.19 & 115.93 & $<5$ & 10 & 9 & 600 & 83 & 3.2 & 55 & 17 & 60 & 8 & 1.1 & 2.82 & 2.7 \\
\hline $13 \mathrm{H}-3$ & $30-60$ & 116.49 & 116.18 & $<5$ & 7 & $<5$ & 600 & 80 & 4.3 & 49 & 15 & 59 & 8 & 0.4 & 2.57 & 1.8 \\
\hline $13 \mathrm{H}-3$ & $60-90$ & 116.79 & 116.43 & $<5$ & 9 & 11 & 700 & 85 & 5.0 & 58 & 17 & 63 & 7 & 0.6 & 2.87 & 2.3 \\
\hline $13 \mathrm{H}-3$ & $90-120$ & 117.09 & 116.69 & $<5$ & 7 & $<5$ & 700 & 77 & 3.1 & 56 & 15 & 59 & 9 & 0.8 & 2.68 & 2.9 \\
\hline $13 \mathrm{H}-3$ & $120-150$ & 117.39 & 116.94 & $<5$ & 8 & 5 & 700 & 77 & 2.5 & 59 & 16 & 68 & 8 & 0.4 & 2.80 & 2.7 \\
\hline $13 \mathrm{H}-4$ & $0-30$ & 117.69 & 117.20 & $<5$ & 8 & $<5$ & 700 & 78 & 3.0 & 61 & 17 & 77 & 9 & 0.9 & 2.95 & 2.6 \\
\hline $13 \mathrm{H}-4$ & $30-60$ & 117.99 & 117.45 & $<5$ & 8 & 6 & 800 & 75 & 2.6 & 62 & 17 & 71 & 9 & 0.9 & 3.04 & 2.9 \\
\hline $13 \mathrm{H}-4$ & $60-90$ & 118.29 & 117.70 & $<5$ & 12 & $<5$ & 700 & 76 & 3.0 & 61 & 16 & 75 & 9 & 0.9 & 3.14 & 2.9 \\
\hline $13 \mathrm{H}-4$ & $90-120$ & 118.59 & 117.96 & $<5$ & 9 & $<5$ & 800 & 79 & 3.3 & 61 & 18 & 65 & 8 & 0.8 & 2.91 & 2.9 \\
\hline $13 \mathrm{H}-4$ & $120-150$ & 118.89 & 118.21 & $<5$ & 9 & 5 & 700 & 84 & 2.0 & 57 & 18 & 63 & 8 & 0.8 & 2.99 & 3.3 \\
\hline $13 \mathrm{H}-5$ & $0-30$ & 119.19 & 118.47 & $<5$ & 8 & 8 & 700 & 81 & 1.5 & 60 & 17 & 68 & 8 & 0.7 & 2.93 & 3.1 \\
\hline $13 \mathrm{H}-5$ & $30-60$ & 119.49 & 118.72 & $<5$ & 10 & 7 & 700 & 77 & 2.2 & 63 & 17 & 66 & 8 & 0.8 & 3.02 & 3.1 \\
\hline $13 \mathrm{H}-5$ & $60-90$ & 119.79 & 118.97 & $<5$ & 12 & $<5$ & 700 & 74 & $<0.8$ & 71 & 18 & 76 & 10 & 0.9 & 3.30 & 3.4 \\
\hline $13 \mathrm{H}-5$ & $90-120$ & 120.09 & 119.23 & $<5$ & 15 & $<5$ & 700 & 72 & 1.1 & 63 & 17 & 74 & 9 & 1.0 & 3.24 & 3.0 \\
\hline $13 \mathrm{H}-5$ & $120-150$ & 120.39 & 119.48 & $<5$ & 15 & 9 & 600 & 59 & 2.0 & 61 & 14 & 71 & 9 & 1.1 & 2.98 & 3.2 \\
\hline $13 \mathrm{H}-6$ & $0-30$ & 120.69 & 119.74 & $<5$ & 16 & 6 & 700 & 43 & 1.8 & 78 & 17 & 82 & 11 & 1.0 & 3.32 & 3.4 \\
\hline $13 \mathrm{H}-6$ & $30-60$ & 120.99 & 119.99 & $<5$ & 16 & $<5$ & 700 & 49 & 2.6 & 67 & 16 & 77 & 10 & 1.2 & 3.19 & 3.0 \\
\hline $13 \mathrm{H}-6$ & $60-90$ & 121.29 & 120.24 & $<5$ & 13 & $<5$ & 700 & 71 & 3.3 & 58 & 15 & 73 & 10 & 0.9 & 2.97 & 2.9 \\
\hline $13 \mathrm{H}-6$ & $90-120$ & 121.59 & 120.50 & $<5$ & 11 & $<5$ & 700 & 68 & 2,1 & 63 & 15 & 69 & 10 & 1.1 & 3.06 & 2.9 \\
\hline $13 \mathrm{H}-6$ & $120-150$ & 121.89 & 120.75 & $<5$ & 8 & $<5$ & 600 & 70 & 2.7 & 58 & 13 & 62 & 9 & 0.9 & 2.83 & 2.8 \\
\hline $13 \mathrm{H}-7$ & $0-30$ & 122.19 & 121.00 & $<5$ & 12 & $<5$ & 700 & 58 & 2.3 & 69 & 16 & 72 & 10 & 0.7 & 3.26 & 3.2 \\
\hline $13 \mathrm{H}-7$ & $30-60$ & 122.49 & 121.26 & $<5$ & 10 & 5 & 700 & 63 & 2.7 & 73 & 16 & 81 & 10 & 1.0 & 3.38 & 3.6 \\
\hline $13 \mathrm{H}-7$ & $60-90$ & 122.79 & 121.51 & $<5$ & 8 & 5 & 700 & 79 & 4.4 & 62 & 15 & 69 & 9 & 0.9 & 3.02 & 2.7 \\
\hline $13 \mathrm{H}-7$ & $90-120$ & 123.09 & 121.76 & $<5$ & 8 & $<5$ & 700 & 89 & 3.9 & 59 & 15 & 66 & 7 & 1.0 & 2.86 & 3.3 \\
\hline $13 \mathrm{H}-8$ & $0-30$ & 123.69 & 122.27 & $<5$ & 14 & 9 & 800 & 80 & 1.8 & 66 & 16 & 79 & 9 & 1.0 & 3.26 & 3.3 \\
\hline $13 \mathrm{H}-8$ & $30-60$ & 123.99 & 122.53 & $<5$ & 10 & 6 & 800 & 92 & 1.0 & 70 & 16 & 66 & 8 & 0.9 & 3.04 & 3.1 \\
\hline $13 \mathrm{H}-8$ & $60-90$ & 124.29 & 122.78 & $<5$ & 8 & 14 & 800 & 96 & 1.8 & 54 & 13 & 63 & 7 & 0.4 & 2.64 & 2.9 \\
\hline $13 \mathrm{H}-\mathrm{CC}$ & $0-22$ & 124.61 & 123.05 & $<5$ & 8 & 9 & 800 & 97 & 1.3 & 63 & 14 & 56 & 7 & 1.1 & 2.77 & 3.8 \\
\hline $14 \mathrm{H}-1$ & $30-60$ & 123.50 & 123.60 & $<5$ & 13 & 8 & 700 & 99 & 2.6 & 65 & 15 & 74 & 8 & 0.7 & 3.18 & 3.0 \\
\hline $14 \mathrm{H}-1 \mathrm{~A}$ & $60-90$ & 123.80 & 123.85 & 6 & 13 & 10 & 800 & 94 & 2.5 & 73 & 17 & 80 & 10 & 1.0 & 3.34 & 3.4 \\
\hline $14 \mathrm{H}-1 \mathrm{~A}$ & $90-120$ & 124.10 & 124.11 & $<5$ & 14 & $<5$ & 700 & 78 & 1.1 & 70 & 17 & 73 & 9 & 1.1 & 3.30 & 3.7 \\
\hline $14 \mathrm{H}-2 \mathrm{~A}$ & $0-40$ & 124.40 & 124.36 & $<5$ & 15 & $<5$ & 700 & 73 & 4.4 & 63 & 15 & 66 & 9 & 0.7 & 3.10 & 3.6 \\
\hline $14 \mathrm{H}-2$ & $40-90$ & 124.80 & 124.69 & $<5$ & 11 & $<5$ & 700 & 87 & 5.8 & 52 & 13 & 61 & 8 & 0.7 & 2.75 & 2.7 \\
\hline $14 \mathrm{H}-2$ & $120-150$ & 125.60 & 125.37 & $<5$ & 13 & 5 & 700 & 68 & 5.7 & 61 & 16 & 62 & 9 & 0.6 & 3.16 & 3.5 \\
\hline $14 \mathrm{H}-3$ & $75-108$ & 126.65 & 126.25 & $<5$ & 9 & 5 & 700 & 69 & 5.5 & 60 & 14 & 65 & 9 & 0.8 & 3.02 & 2.8 \\
\hline $14 \mathrm{H}-3$ & $116-150$ & 127.06 & 126.59 & $<5$ & 11 & $<5$ & 700 & 79 & 4.9 & 67 & 14 & 71 & 9 & 0.8 & 3.14 & 3.6 \\
\hline $14 \mathrm{H}-4$ & $0-30$ & 127.40 & 126.88 & $<5$ & 13 & 15 & 900 & 99 & 5.1 & 76 & 16 & 79 & 10 & 0.7 & 3.65 & 3.7 \\
\hline $14 \mathrm{H}-4$ & $30-60$ & 127.70 & 127.13 & $<5$ & 17 & $<5$ & 800 & 76 & 5.0 & 65 & 15 & 73 & 9 & 0.6 & 3.34 & 3.9 \\
\hline $14 \mathrm{H}-4$ & $90-120$ & 128.30 & 127.63 & $<5$ & 8 & $<5$ & 800 & 100 & 4.1 & 53 & 13 & 59 & 8 & 0.9 & 2.74 & 3.2 \\
\hline $14 \mathrm{H}-5 \mathrm{~A}$ & $0-30$ & 128.90 & 128.13 & $<5$ & 9 & 5 & 700 & 92 & 5.4 & 57 & 13 & 65 & 8 & 0.7 & 2.95 & 3.5 \\
\hline
\end{tabular}


Table 1 (continued).

\begin{tabular}{|c|c|c|c|c|c|c|c|c|c|c|c|c|c|c|c|c|}
\hline $\begin{array}{l}\text { Core, } \\
\text { section }\end{array}$ & $\begin{array}{c}\text { Interval } \\
(\mathrm{cm})\end{array}$ & $\begin{array}{c}\text { ODP } \\
\text { depth } \\
\text { (mbsf) }\end{array}$ & $\begin{array}{l}\text { Corrected } \\
\text { depth } \\
\text { (mbsf) }\end{array}$ & $\underset{(\mathrm{ppm})}{\mathrm{Ag}}$ & $\begin{array}{c}\text { As } \\
(\mathrm{ppm})\end{array}$ & $\begin{array}{c}\mathrm{Au} \\
(\mathrm{ppb})\end{array}$ & $\begin{array}{c}\mathrm{Ba} \\
(\mathrm{ppm})\end{array}$ & $\begin{array}{c}\mathrm{Br} \\
(\mathrm{ppm})\end{array}$ & $\begin{array}{l}\mathrm{Ca} \\
(\%)\end{array}$ & $\begin{array}{c}\mathrm{Ce} \\
(\mathrm{ppm})\end{array}$ & $\begin{array}{c}\mathrm{Co} \\
(\mathrm{ppm})\end{array}$ & $\underset{(\mathrm{ppm})}{\mathrm{Cr}}$ & $\begin{array}{c}\mathrm{Cs} \\
(\mathrm{ppm})\end{array}$ & $\begin{array}{c}\mathrm{Eu} \\
(\mathrm{ppm})\end{array}$ & $\begin{array}{l}\mathrm{Fe} \\
(\%)\end{array}$ & $\underset{(\mathrm{ppm})}{\mathrm{Hf}}$ \\
\hline $14 \mathrm{H}-5$ & $60-90$ & 129.50 & 128.64 & $<5$ & 6 & 5 & 700 & 100 & 3.9 & 48 & 12 & 51 & 7 & 0.7 & 2.46 & 2.5 \\
\hline $14 \mathrm{H}-5$ & $90-120$ & 129.80 & 128.89 & $<5$ & 8 & $<5$ & 700 & 83 & 3.3 & 43 & 12 & 42 & 5 & 0.6 & 2.47 & 2.6 \\
\hline $14 \mathrm{H}-5$ & $120-150$ & 130.10 & 129.14 & $<5$ & 9 & 69 & 700 & 98 & 3.2 & 48 & 14 & 57 & 6 & 0.7 & 2.58 & 2.8 \\
\hline $14 \mathrm{H}-6$ & $0-30$ & 130.40 & 129.39 & $<5$ & 9 & $<5$ & 800 & 110 & 3.2 & 57 & 15 & 67 & 8 & 1.0 & 2.98 & 2.7 \\
\hline $14 \mathrm{H}-6$ & $60-90$ & 131.00 & 129.90 & $<5$ & 10 & 6 & 800 & 110 & 1.7 & 71 & 16 & 82 & 9 & 0.9 & 3.43 & 3.9 \\
\hline $14 \mathrm{H}-6$ & $90-120$ & 131.30 & 130.15 & $<5$ & 15 & 7 & 900 & 100 & 0.9 & 76 & 18 & 85 & 10 & 0.9 & 3.50 & 4.1 \\
\hline $14 \mathrm{H}-6$ & $120-150$ & 131.60 & 130.40 & $<5$ & 12 & 6 & 900 & 100 & 0.8 & 73 & 18 & 82 & II & 0.8 & 3.50 & 3.5 \\
\hline $14 \mathrm{H}-7$ & $0-30$ & 131.90 & 130.65 & $<5$ & 9 & $<5$ & 900 & 94 & 3.0 & 72 & 17. & 78 & 10 & 0.7 & 3.41 & 3.3 \\
\hline $14 \mathrm{H}-7$ & $30-60$ & 132.20 & 130.90 & $<5$ & 10 & 5 & 800 & 71 & 3.5 & 70 & 18 & 77 & 11 & 1.0 & 3.62 & 3.9 \\
\hline $14 \mathrm{H}-7$ & $60-97$ & 132.50 & 131.16 & $<5$ & 8 & 6 & 700 & 61 & 4.2 & 69 & 17 & 71 & 9 & 0.8 & 3.38 & 3.3 \\
\hline $14 \mathrm{H}-7$ & $137-150$ & 133.20 & 131.74 & $<5$ & 7 & 5 & 700 & 56 & 2.5 & 70 & 16 & 71 & 10 & 0.9 & 3.47 & 3.3 \\
\hline $14 \mathrm{H}-8$ & $0-30$ & 133.50 & 131.99 & $<5$ & 8 & 11 & 700 & 61 & 2.7 & 73 & 19 & 75 & 10 & 0.9 & 3.66 & 3.1 \\
\hline $14 \mathrm{H}-8$ & $30-60$ & 133.80 & 132.25 & $<5$ & 9 & $<5$ & 800 & 61 & 1.7 & 73 & 19 & 84 & 10 & 0.8 & 3.75 & 3.3 \\
\hline $14 \mathrm{H}-8$ & $60-90$ & 134.10 & 132.50 & $<5$ & 11 & 5 & 800 & 60 & 1.8 & 74 & 19 & 84 & 12 & 1.1 & 3.78 & 3.7 \\
\hline $14 \mathrm{H}-8$ & $90-96$ & 134.40 & 132.75 & $<5$ & 12 & $<5$ & 700 & 67 & 2.0 & 70 & 19 & 86 & 10 & 1.0 & 3.83 & 3.2 \\
\hline $15 \mathrm{H}-1$ & $0-30$ & 132.90 & 133.05 & $<5$ & 8 & 7 & 800 & 88 & 0.9 & 61 & 16 & 64 & 8 & 0.7 & 3.10 & 2.8 \\
\hline $15 \mathrm{H}-1$ & $60-90$ & 133.50 & 133.56 & $<5$ & 8 & 16 & 800 & 77 & 1.5 & 58 & 16 & 72 & 9 & 0.9 & 2.99 & 3.2 \\
\hline $15 \mathrm{H}-1$ & $90-116$ & 133.80 & 133.81 & $<5$ & 8 & 7 & 800 & 81 & 2.4 & 61 & 17 & 69 & 9 & 0.7 & 3.19 & 3.0 \\
\hline $15 \mathrm{H}-2$ & $0-30$ & 134.06 & 134.03 & $<5$ & 14 & 8 & 1000 & 95 & 0.8 & 68 & 18 & 80 & 10 & 0.8 & 3.41 & 3.0 \\
\hline $15 \mathrm{H}-2$ & $30-80$ & 134.36 & 134.29 & $<5$ & 20 & $<5$ & 1000 & 88 & 0.6 & 65 & 17 & 79 & 11 & 1.0 & 3.39 & 2.6 \\
\hline $15 \mathrm{H}-2$ & $80-120$ & 134.86 & 134.71 & $<5$ & 17 & 5 & 800 & 87 & 0.5 & 63 & 17 & 82 & 11 & 0.9 & 3.37 & 3.1 \\
\hline $15 \mathrm{H}-2$ & $120-150$ & 135.26 & 135.05 & $<5$ & 12 & 6 & 800 & 84 & 2.0 & 55 & 15 & 70 & 10 & 0.7 & 2.99 & 2.5 \\
\hline $15 \mathrm{H}-3$ & $0-30$ & 135.56 & 135.31 & $<5$ & 14 & $<5$ & 700 & 74 & 3.7 & 60 & 14 & 68 & 10 & 1.0 & 3.01 & 3.2 \\
\hline $15 \mathrm{H}-3$ & $30-60$ & 135.86 & 135.56 & $<5$ & 14 & $<5$ & 800 & 63 & 2.4 & 69 & 15 & 80 & 11 & 0.6 & 3.39 & 3.5 \\
\hline $15 \mathrm{H}-3$ & $60-90$ & 136.16 & 135.81 & $<5$ & 10 & 7 & 800 & 67 & 3.3 & 69 & 16 & 78 & 10 & 0.6 & 3.29 & 2.9 \\
\hline $15 \mathrm{H}-3$ & $90-120$ & 136.46 & 136.07 & $<5$ & 11 & 5 & 800 & 68 & 2.5 & 67 & 16 & 80 & 11 & 1.0 & 3.27 & 3.1 \\
\hline $15 \mathrm{H}-3$ & $120-150$ & 136.76 & 136.32 & $<5$ & 12 & $<5$ & 800 & 74 & 3.1 & 58 & 16 & 73 & 10 & 0.9 & 3.07 & 2.3 \\
\hline $15 \mathrm{H}-4$ & $0-30$ & 137.06 & 136.58 & $<5$ & 13 & $<5$ & 900 & 85 & 3.8 & 59 & 13 & 73 & 9 & 0.8 & 2.99 & 3.2 \\
\hline $15 \mathrm{H}-4$ & $30-60$ & 137.36 & 136.83 & $<5$ & 15 & $<5$ & 800 & 70 & 3.8 & 66 & 15 & 81 & 10 & 0.9 & 3.39 & 3.4 \\
\hline $15 \mathrm{H}-4$ & $60-90$ & 137.66 & 137.09 & $<5$ & 14 & $<5$ & 700 & 72 & 2.1 & 65 & 15 & 82 & 10 & 0.8 & 3.30 & 3.0 \\
\hline $15 \mathrm{H}-4$ & $90-120$ & 137.96 & 137.34 & $<5$ & 11 & 10 & 700 & 86 & 1.6 & 61 & 15 & 75 & 10 & 0.7 & 3.16 & 3.2 \\
\hline $15 \mathrm{H}-4$ & $120-150$ & 138.26 & 137.59 & $<5$ & 10 & 9 & 700 & 96 & 2.3 & 55 & 15 & 70 & 10 & 0.7 & 3.00 & 3.1 \\
\hline $15 \mathrm{H}-5$ & $0-30$ & 138.56 & 137.85 & $<5$ & 10 & 8 & 700 & 100 & 2.7 & 47 & 16 & 62 & 9 & 0.5 & 2.91 & 2.8 \\
\hline $15 \mathrm{H}-5$ & $30-60$ & 138.86 & 138.10 & $<5$ & 9 & 17 & 600 & 100 & 3.4 & 60 & 17 & 69 & 8 & 0.6 & 2.87 & 3.0 \\
\hline $15 \mathrm{H}-5$ & $60-90$ & 139.16 & 138.36 & $<5$ & 7 & 7 & 700 & 94 & 4.1 & 48 & 13 & 58 & 8 & 0.8 & 2.48 & 2.4 \\
\hline $15 \mathrm{H}-5$ & $90-117$ & 139.46 & 138.61 & $<5$ & 7 & 5 & 700 & 97 & 4.9 & 47 & 14 & 60 & 7 & 0.9 & 2.60 & 2.7 \\
\hline $15 \mathrm{H}-6$ & $0-30$ & 140.06 & 139.12 & $<5$ & 8 & 5 & 600 & 91 & 3.0 & 52 & 14 & 64 & 8 & 0.8 & 2.77 & 2.9 \\
\hline $15 \mathrm{H}-6$ & $30-60$ & 140.36 & 139.37 & $<5$ & 9 & 6 & 700 & 90 & 2.7 & 57 & 15 & 74 & 9 & 1.0 & 2.99 & 2.9 \\
\hline $15 \mathrm{H}-6$ & $60-90$ & 140.66 & 139.63 & $<5$ & 9 & $<5$ & 700 & 85 & 2.8 & 57 & 15 & 74 & 9 & 0.9 & 3.03 & 2.6 \\
\hline $15 \mathrm{H}-6$ & $90-120$ & 140.96 & 139.88 & $<5$ & 10 & $<5$ & 800 & 90 & 2.6 & 64 & 16 & 82 & 11 & 1.1 & 3.38 & 3.3 \\
\hline $15 \mathrm{H}-6$ & $120-150$ & 141.26 & 140.14 & $<5$ & 15 & 12 & 800 & 92 & 4.0 & 54 & 16 & 76 & 9 & 0.6 & 2.99 & 2.5 \\
\hline $15 \mathrm{H}-7$ & $30-53$ & 141.86 & 140.65 & $<5$ & 11 & 5 & 800 & 88 & 4.6 & 48 & 13 & 56 & 8 & 0.8 & 2.32 & 2.3 \\
\hline $15 \mathrm{H}-7$ & $53-90$ & 142.09 & 140.84 & $<5$ & 15 & 6 & 800 & 69 & 2.6 & 56 & 15 & 67 & 9 & 0.8 & 2.91 & 3.2 \\
\hline $15 \mathrm{H}-7$ & $90-120$ & 142.46 & 141.15 & $<5$ & 15 & 7 & 900 & 66 & 0.9 & 70 & 17 & 87 & 12 & 1.1 & 3.66 & 3.6 \\
\hline $15 \mathrm{H}-7$ & $120-150$ & 142.76 & 141.41 & $<5$ & 14 & 13 & 900 & 70 & 1.5 & 63 & 15 & 88 & 12 & 0.8 & 3.42 & 3.5 \\
\hline $15 \mathrm{H}-8$ & $0-30$ & 143.06 & 141.66 & $<5$ & 11 & 11 & 800 & 64 & 2.0 & 67 & 14 & 78 & 11 & 0.9 & 3.23 & 3.5 \\
\hline $15 \mathrm{H}-8$ & $30-60$ & 143.36 & 141.92 & $<5$ & 11 & $<5$ & 800 & 66 & 3.8 & 63 & 15 & 71 & 10 & 0.9 & 3.03 & 2.8 \\
\hline $15 \mathrm{H}-8$ & $60-90$ & 143.66 & 142.17 & $<5$ & 12 & 8 & 700 & 78 & 2.1 & 55 & 14 & 82 & 9 & 0.6 & 3.12 & 2.9 \\
\hline $15 \mathrm{H}-\mathrm{CC}$ & $0-27$ & 143.99 & 142.45 & $<5$ & 10 & $<5$ & 800 & 83 & 2.3 & 62 & 14 & 79 & 9 & 0.8 & 3.20 & 3.3 \\
\hline
\end{tabular}


Table 1 (continued).

\begin{tabular}{|c|c|c|c|c|c|c|c|c|c|c|c|c|c|c|c|c|c|}
\hline $\begin{array}{c}\text { Core, } \\
\text { section }\end{array}$ & $\underset{(\mathrm{ppm})}{\mathrm{La}}$ & $\underset{(\mathrm{ppm})}{\mathrm{Lu}}$ & $\begin{array}{c}\text { Mo } \\
(\mathrm{ppm})\end{array}$ & $\begin{array}{l}\mathrm{Na} \\
(\%)\end{array}$ & $\underset{(\mathrm{ppm})}{\mathrm{Nd}}$ & $\begin{array}{c}\mathrm{Rb} \\
(\mathrm{ppm})\end{array}$ & $\begin{array}{c}\mathrm{Sb} \\
(\mathrm{ppm})\end{array}$ & $\underset{(\mathrm{ppm})}{\mathrm{Sc}}$ & $\underset{(\mathrm{ppm})}{\mathrm{Se}}$ & $\underset{(\mathrm{ppm})}{\mathrm{Sm}}$ & $\underset{(\mathrm{ppm})}{\mathrm{Ta}}$ & $\begin{array}{c}\mathrm{Tb} \\
\text { (ppm) }\end{array}$ & $\begin{array}{c}\text { Th } \\
(\mathrm{ppm})\end{array}$ & $\underset{(\mathrm{ppm})}{\mathrm{U}}$ & $\begin{array}{c}\mathrm{W} \\
(\mathrm{ppm})\end{array}$ & $\begin{array}{c}\mathrm{Yb} \\
(\mathrm{ppm})\end{array}$ & $\underset{(\mathrm{ppm})}{\mathrm{Zn}}$ \\
\hline $13 \mathrm{H}-1$ & 40.3 & 0.38 & 7 & 2.0 & 33 & 160 & 3.5 & 16.4 & $<3$ & 6.1 & $<1$ & 0.7 & 15.0 & 4.8 & $<3$ & 2.5 & 130 \\
\hline $13 \mathrm{H}-1$ & 37.2 & 0.35 & 19 & 1.9 & 30 & 110 & 2.2 & 14.1 & $<3$ & 5.4 & $<1$ & 0.6 & 13.0 & 8.7 & 3 & 2.3 & 130 \\
\hline $13 \mathrm{H}-1$ & 33.3 & 0.36 & 47 & 1.9 & 27 & 120 & 2.3 & 14.1 & $<3$ & 5.0 & $<1$ & 0.6 & 12.0 & 11.7 & $<3$ & 2.4 & 110 \\
\hline $13 \mathrm{H}-1$ & 32.6 & 0.35 & 10 & 1.5 & 27 & 110 & 2.8 & 12.8 & $<3$ & 4.5 & 1 & 0.6 & 12.0 & 3.8 & $<3$ & 2.3 & 80 \\
\hline $13 \mathrm{H}-2$ & 32.8 & 0.35 & 6 & 1.7 & 26 & 130 & 2.3 & 13.3 & $<3$ & 4.4 & $i$ & 0.7 & 12.0 & 4.6 & $<3$ & 2.3 & 90 \\
\hline $13 \mathrm{H}-2$ & 29.7 & 0.30 & 6 & 1.9 & 25 & 100 & 1.4 & 12.2 & $<3$ & 4.4 & $i$ & 0.6 & 11.0 & 6.2 & $<3$ & 1.9 & 80 \\
\hline $13 \mathrm{H}-2$ & 29.4 & 0.30 & 8 & 1.8 & 25 & 100 & 1.5 & 12.1 & $<3$ & 4.4 & $<1$ & 0.6 & 12.0 & 8.3 & $<3$ & 1.9 & 170 \\
\hline $13 \mathrm{H}-2$ & 30.1 & 0.32 & 5 & 1.7 & 23 & 110 & 1.8 & 12.6 & $<3$ & 4.1 & $<1$ & 0.6 & 12.0 & 5.5 & $<3$ & 2.1 & 90 \\
\hline $13 \mathrm{H}-2$ & 28.4 & 0.29 & 6 & 1.7 & 27 & 100 & 1.4 & 11.6 & $<3$ & 3.9 & 1 & 0.7 & 11.0 & 4.0 & $<3$ & 1.9 & 120 \\
\hline $13 \mathrm{H}-3$ & 25.1 & 0.31 & 6 & 1.7 & 25 & 60 & 1.3 & 11.1 & $<3$ & 3.5 & $<1$ & 0.6 & 9.1 & 5.4 & $<3$ & 2.0 & 70 \\
\hline $13 \mathrm{H}-3$ & 23.1 & 0.26 & 6 & 1.6 & 18 & 90 & 1.1 & 10.4 & $<3$ & 3.2 & $<1$ & 0.5 & 8.1 & 4.6 & $<3$ & 1.7 & 110 \\
\hline $13 \mathrm{H}-3$ & 25.0 & 0.28 & 7 & 1.8 & 20 & 80 & 1.2 & 10.9 & $<3$ & 3.5 & $<1$ & 0.5 & 9.3 & 5.4 & 3 & 1.8 & 80 \\
\hline $13 \mathrm{H}-3$ & 26.6 & 0.27 & $<5$ & 1.7 & 23 & 70 & 1.3 & 10.1 & $<3$ & 3.4 & $<1$ & $<0.5$ & 8.6 & 4.1 & $<3$ & 1.7 & 110 \\
\hline $13 \mathrm{H}-3$ & 27.7 & 0.26 & 6 & 1.7 & 24 & 90 & 1.3 & 10.7 & $<3$ & 3.7 & $<1$ & 0.5 & 9.3 & 3.6 & $<3$ & 1.7 & 120 \\
\hline $13 \mathrm{H}-4$ & 28.4 & 0.29 & 5 & 1.7 & 20 & 100 & 1.5 & 11.5 & $<3$ & 3.9 & $<1$ & 0.5 & 10.0 & 4.7 & $<3$ & 1.9 & 130 \\
\hline $13 \mathrm{H}-4$ & 29.0 & 0.30 & 5 & 1.7 & 21 & 110 & 1.5 & 11.5 & $<3$ & 4.3 & $<1$ & 0.6 & 11.0 & 5.0 & $<3$ & 1.9 & 120 \\
\hline $13 \mathrm{H}-4$ & 30.9 & 0.32 & 5 & 1.7 & 21 & 120 & 1.5 & 12.6 & $<3$ & 4.2 & $<1$ & 0.6 & 11.0 & 5.3 & $<3$ & 2.1 & 110 \\
\hline $13 \mathrm{H}-4$ & 27.7 & 0.31 & 6 & 1.7 & 23 & 120 & 1.3 & 12.0 & $<3$ & 3.8 & $<1$ & 0.5 & 11.0 & 5.4 & $<3$ & 2.0 & 120 \\
\hline $13 \mathrm{H}-4$ & 27.6 & 0.29 & 8 & 1.8 & 25 & 80 & 1.5 & 11.2 & $<3$ & 4.2 & 1 & $<0.5$ & 11.0 & 5.6 & $<3$ & 1.8 & 90 \\
\hline $13 \mathrm{H}-5$ & 28.7 & 0.30 & 9 & 1.8 & 25 & 110 & 1.3 & 11.3 & $<3$ & 3.8 & 1 & 0.5 & 10.0 & 5.0 & $<3$ & 2.0 & 90 \\
\hline $13 \mathrm{H}-5$ & 28.9 & 0.31 & 11 & 1.7 & 25 & 90 & 1.9 & 11.6 & $<3$ & 3.9 & i & 0.5 & 11.0 & 5.0 & $<3$ & 2.0 & 100 \\
\hline $13 \mathrm{H}-5$ & 32.3 & 0.31 & 14 & 1.7 & 27 & 120 & 1.8 & 13.2 & $<3$ & 4.4 & $<1$ & 0.5 & 11.0 & 6.7 & $<3$ & 2.0 & 90 \\
\hline $13 \mathrm{H}-5$ & 30.4 & 0.30 & 49 & 1.6 & 21 & 100 & 1.9 & 12.3 & $<3$ & 4.2 & $<1$ & 0.6 & 10.0 & 11.2 & 3 & 2.0 & 120 \\
\hline $13 \mathrm{H}-5$ & 31.5 & 0.33 & 40 & 1.5 & 25 & 130 & 1.9 & 11.9 & $<3$ & 4.3 & $<1$ & 0.6 & 10.0 & 11.5 & $<3$ & 2.2 & 110 \\
\hline $13 \mathrm{H}-6$ & 36.6 & 0.34 & 25 & 1.5 & 25 & 130 & 3.1 & 12.9 & $<3$ & 5.0 & $<1$ & 1.0 & 11.0 & 6.1 & $<3$ & 2.3 & 70 \\
\hline $13 \mathrm{H}-6$ & 33.1 & 0.34 & 20 & 1.5 & 24 & 120 & 2.4 & 12.4 & $<3$ & 4.5 & $<1$ & 1.1 & 11.0 & 5.0 & $<3$ & 2.4 & 100 \\
\hline $13 \mathrm{H}-6$ & 29.3 & 0.32 & 22 & 1.6 & 23 & 100 & 1.9 & 12.3 & $<3$ & 4.0 & $<1$ & 0.8 & 10.0 & 6.2 & $<3$ & 2.1 & 90 \\
\hline $13 \mathrm{H}-6$ & 30.7 & 0.31 & 8 & 1.6 & 24 & 90 & 2.2 & 12.1 & $<3$ & 3.9 & 1 & 0.5 & 10.0 & 4.2 & $<3$ & 2.0 & 110 \\
\hline $13 \mathrm{H}-6$ & 27.7 & 0.26 & 10 & 1.5 & 21 & 90 & 1.4 & 11.9 & $<3$ & 3.7 & 1 & 0.5 & 9.6 & 5.6 & $<3$ & 1.7 & 120 \\
\hline $13 \mathrm{H}-7$ & 32.1 & 0.34 & 5 & 1.5 & 23 & 130 & 2.3 & 12.8 & $<3$ & 4.2 & 1 & 0.6 & 11.0 & 3.5 & $<3$ & 2.2 & 100 \\
\hline $13 \mathrm{H}-7$ & 34.0 & 0.32 & 7 & 1.7 & 29 & 120 & 1.7 & 13.3 & $<3$ & 4.4 & 1 & 0.8 & 12.0 & 3.8 & $<3$ & 2.1 & 70 \\
\hline $13 \mathrm{H}-7$ & 28.8 & 0.32 & 5 & 1.7 & 20 & 110 & 1.2 & 12.0 & $<3$ & 3.8 & $<1$ & 0.7 & 10.0 & 4.6 & $<3$ & 2.1 & 100 \\
\hline $13 \mathrm{H}-7$ & 28.1 & 0.29 & 8 & 1.7 & 20 & 90 & 1.0 & 11.4 & $<3$ & 3.7 & 1 & 0.6 & 9.7 & 4.9 & $<3$ & 1.9 & 130 \\
\hline $13 \mathrm{H}-8$ & 31.8 & 0.32 & 12 & 1.8 & 21 & 110 & 1.9 & 12.3 & $<3$ & 4.1 & 1 & 0.5 & 11.0 & 4.5 & $<3$ & 2.1 & 120 \\
\hline $13 \mathrm{H}-8$ & 28.5 & 0.29 & 22 & 1.8 & 22 & 70 & 1.1 & 10.5 & $<3$ & 3.7 & $i$ & 0.6 & 9.7 & 4.6 & $<3$ & 1.9 & 130 \\
\hline $13 \mathrm{H}-8$ & 25.5 & 0.27 & 14 & 1.8 & 24 & 100 & 1.0 & 10.3 & $<3$ & 3.4 & $<1$ & 0.6 & 8.3 & 6.4 & $<3$ & 1.8 & 100 \\
\hline $13 \mathrm{H}-\mathrm{CC}$ & 32.3 & 0.29 & 11 & 1.9 & 22 & 90 & 1.0 & 9.3 & $<3$ & 3.6 & 1 & 0.6 & 10.0 & 6.0 & $<3$ & 1.9 & 80 \\
\hline $14 \mathrm{H}-1$ & 29.6 & 0.32 & 15 & 1.8 & 22 & 110 & 1.4 & 12.4 & $<3$ & 4.0 & 1 & 0.7 & 10.0 & 10.6 & $<3$ & 2.1 & 100 \\
\hline $14 \mathrm{H}-1 \mathrm{~A}$ & 34.7 & 0.34 & 23 & 1.8 & 24 & 100 & 1.5 & 12.7 & $<3$ & 4.6 & $i$ & 0.8 & 12.0 & 10.3 & 3 & 2.2 & 170 \\
\hline $14 \mathrm{H}-1 \mathrm{~A}$ & 34.1 & 0.33 & 25 & 1.7 & 21 & 100 & 1.8 & 11.8 & $<3$ & 4.4 & 1 & 0.8 & 11.0 & 7.8 & $<3$ & 2.2 & 100 \\
\hline $14 \mathrm{H}-2 \mathrm{~A}$ & 31.4 & 0.30 & 15 & 1.6 & 24 & 90 & 2.3 & 11.1 & $<3$ & 4.0 & 1 & 0.7 & 10.0 & 5.3 & $<3$ & 2.0 & 100 \\
\hline $14 \mathrm{H}-2$ & 25.6 & 0.25 & 23 & 1.7 & 22 & 60 & 1.3 & 10.3 & $<3$ & 3.4 & $<1$ & 0.7 & 8.7 & 6.6 & $<3$ & 1.7 & 60 \\
\hline $14 \mathrm{H}-2$ & 29.8 & 0.27 & 10 & 1.6 & 19 & 110 & 1.7 & 11.4 & $<3$ & 4.0 & $<1$ & 0.7 & 10.0 & 3.7 & 3 & 1.8 & 60 \\
\hline $14 \mathrm{H}-3$ & 29.1 & 0.29 & 8 & 1.6 & 19 & 100 & 1.7 & 11.8 & $<3$ & 3.9 & $<1$ & 0.7 & 9.9 & 4.4 & $<3$ & 1.9 & 100 \\
\hline $14 \mathrm{H}-3$ & 29.8 & 0.31 & 10 & 1.7 & 21 & 100 & 1.7 & 12.0 & $<3$ & 4.0 & $<1$ & 0.7 & 10.0 & 5.2 & $<3$ & 2.0 & 90 \\
\hline $14 \mathrm{H}-4$ & 34.6 & 0.34 & 9 & 1.9 & 34 & 120 & 2.2 & 14.0 & $<3$ & 5.5 & $<1$ & 1.0 & 12.0 & 7.7 & $<3$ & 2.3 & 110 \\
\hline $14 \mathrm{H}-4$ & 32.1 & 0.33 & 5 & 1.7 & 23 & 100 & 2.5 & 12.6 & $<3$ & 4.3 & $<1$ & 0.7 & 11.0 & 4.3 & $<3$ & 2.2 & 110 \\
\hline $14 \mathrm{H}-4$ & 25.4 & 0.33 & 14 & 1.9 & 22 & 80 & 1.1 & 10.6 & $<3$ & 3.6 & 1 & 0.6 & 8.9 & 6.5 & $<3$ & 2.2 & 90 \\
\hline $14 \mathrm{H}-5 \mathrm{~A}$ & 31.6 & 0.31 & 9 & 1.8 & 23 & 90 & 1.0 & 11.1 & $<3$ & 4.0 & $i$ & 0.5 & 10.0 & 5.2 & $<3$ & 2.0 & 120 \\
\hline
\end{tabular}


Table 1 (continued).

\begin{tabular}{|c|c|c|c|c|c|c|c|c|c|c|c|c|c|c|c|c|c|}
\hline $\begin{array}{l}\text { Core, } \\
\text { section }\end{array}$ & $\begin{array}{c}\mathrm{La} \\
(\mathrm{ppm})\end{array}$ & $\begin{array}{c}\text { Lu } \\
(\mathrm{ppm})\end{array}$ & $\begin{array}{c}\text { Mo } \\
\text { (ppm) }\end{array}$ & $\begin{array}{l}\mathrm{Na} \\
(\%)\end{array}$ & $\begin{array}{l}\text { Nd } \\
(\mathrm{ppm})\end{array}$ & $\begin{array}{c}\mathrm{Rb} \\
(\mathrm{ppm})\end{array}$ & $\begin{array}{c}\mathrm{Sb} \\
(\mathrm{ppm})\end{array}$ & $\underset{(\mathrm{ppm})}{\mathrm{Sc}}$ & $\begin{array}{c}\mathrm{Se} \\
(\mathrm{ppm})\end{array}$ & $\begin{array}{c}\mathrm{Sm} \\
(\mathrm{ppm})\end{array}$ & $\begin{array}{c}\mathrm{Ta} \\
(\mathrm{ppm})\end{array}$ & $\begin{array}{c}\mathrm{Tb} \\
\text { (ppm) }\end{array}$ & $\begin{array}{c}\text { Th } \\
\text { (ppm) }\end{array}$ & $\begin{array}{c}\mathrm{U} \\
(\mathrm{ppm})\end{array}$ & $\begin{array}{c}\text { W } \\
(\mathrm{ppm})\end{array}$ & $\begin{array}{c}\mathrm{Yb} \\
(\mathrm{ppm})\end{array}$ & $\underset{\text { (ppm) }}{\mathrm{Zn}}$ \\
\hline $14 \mathrm{H}-5$ & 23.2 & 0.23 & 10 & 1.9 & 19 & 80 & 0.8 & 9.4 & $<3$ & 3.1 & $<1$ & $<0.5$ & 8.1 & 4.5 & $<3$ & 1.5 & 90 \\
\hline $14 \mathrm{H}-5$ & 21.9 & 0.20 & 7 & 2.4 & 19 & 60 & 1.1 & 9.0 & $<3$ & 2.8 & 1 & $<0.5$ & 6.9 & 3.8 & 3 & 1.3 & 130 \\
\hline $14 \mathrm{H}-5$ & 24.4 & 0.24 & 6 & 1.9 & 24 & 80 & 1.0 & 10.4 & $<3$ & 3.2 & $<1$ & 0.5 & 8.2 & 4.9 & $<3$ & 1.6 & 110 \\
\hline $14 \mathrm{H}-6$ & 27.1 & 0.31 & 8 & 1.9 & 24 & 90 & 1.2 & 11.5 & $<3$ & 3.8 & $<1$ & $<0.5$ & 9.5 & 5.4 & $<3$ & 2.0 & 100 \\
\hline $14 \mathrm{H}-6$ & 33.4 & 0.32 & 10 & 1.9 & 27 & 120 & 1.9 & 12.9 & $<3$ & 4.5 & 1 & 0.6 & 12.0 & 6.9 & 3 & 2.1 & 120 \\
\hline $14 \mathrm{H}-6$ & 35.6 & 0.32 & 17 & 1.9 & 29 & 110 & 1.8 & 13.1 & $<3$ & 4.8 & 1 & 0.6 & 12.0 & 7.3 & 3 & 2.1 & 150 \\
\hline $14 \mathrm{H}-6$ & 33.5 & 0.31 & 16 & 1.9 & 28 & 120 & 1.8 & 13.4 & $<3$ & 4.6 & 1 & 0.8 & 12.0 & 7.0 & 3 & 2.0 & 110 \\
\hline $14 \mathrm{H}-7$ & 32.0 & 0.36 & 13 & 1.7 & 26 & 110 & 1.6 & 13.7 & $<3$ & 4.5 & 1 & 0.8 & 11.0 & 6.6 & $<3$ & 2.4 & 100 \\
\hline $14 \mathrm{H}-7$ & 34.0 & 0.35 & $<5$ & 1.7 & 28 & 130 & 2.0 & 14.2 & $<3$ & 4.9 & $<1$ & 0.9 & 12.0 & 5.0 & 3 & 2.3 & 140 \\
\hline $14 \mathrm{H}-7$ & 33.0 & 0.31 & 8 & 1.6 & 25 & 110 & 2.0 & 13.1 & $<3$ & 4.4 & 1 & 0.7 & 12.0 & 3.9 & 3 & 2.1 & 120 \\
\hline $14 \mathrm{H}-7$ & 33.2 & 0.32 & $<5$ & 1.5 & 21 & 110 & 1.4 & 13.5 & $<3$ & 4.5 & 1 & 0.5 & 12.0 & 3.4 & 3 & 2.1 & 100 \\
\hline $14 \mathrm{H}-8$ & 33.3 & 0.33 & 5 & 1.6 & 19 & 120 & 1.5 & 14.0 & $<3$ & 4.5 & 1 & 0.7 & 12.0 & 3.8 & 4 & 2.2 & 120 \\
\hline $14 \mathrm{H}-8$ & 34.5 & 0.32 & 6 & 1.6 & 26 & 130 & 1.7 & 14.4 & $<3$ & 4.7 & 1 & 0.9 & 12.0 & 4.0 & 3 & 2.1 & 130 \\
\hline $14 \mathrm{H}-8$ & 35.3 & 0.35 & 5 & 1.6 & 28 & 120 & 1.9 & 14.3 & $<3$ & 4.7 & 1 & 0.5 & 13.0 & 3.9 & 3 & 2.3 & 90 \\
\hline $14 \mathrm{H}-8$ & 34.3 & 0.31 & 6 & 1.6 & 24 & 140 & 1.6 & 13.7 & $<3$ & 4.6 & 2 & 0.5 & 12.0 & 4.0 & $<3$ & 2.1 & 110 \\
\hline $15 \mathrm{H}-1$ & 29.1 & 0.30 & 7 & 1.8 & 21 & 110 & 1.1 & 11.8 & $<3$ & 3.9 & $<1$ & 0.6 & 10.0 & 6.1 & $<3$ & 1.9 & 100 \\
\hline $15 \mathrm{H}-1$ & 28.8 & 0.31 & 6 & 1.7 & 21 & 110 & 1.2 & 11.8 & $<3$ & 3.8 & 1 & 0.6 & 10.0 & 4.4 & $<3$ & 2.0 & 110 \\
\hline $15 \mathrm{H}-1$ & 29.5 & 0.30 & 6 & 1.7 & 21 & 100 & 1.2 & 12.3 & $<3$ & 3.9 & $<1$ & 0.7 & 10.0 & 4.6 & $<3$ & 1.9 & 140 \\
\hline $15 \mathrm{H}-2$ & 31.1 & 0.30 & 23 & 1.7 & 27 & 110 & 1.3 & 13.1 & $<3$ & 4.3 & 1 & 0.5 & 11.0 & 10.4 & $<3$ & 2.0 & 110 \\
\hline $15 \mathrm{H}-2$ & 31.3 & 0.32 & 32 & 1.6 & 25 & 110 & 1.6 & 12.8 & $<3$ & 4.5 & 1 & 0.7 & 11.0 & 15.2 & 3 & 2.1 & 110 \\
\hline $15 \mathrm{H}-2$ & 32.7 & 0.34 & 30 & 1.7 & 26 & 120 & 1.4 & 13.4 & $<3$ & 4.7 & $<1$ & 0.6 & 12.0 & 13.3 & 3 & 2.2 & 120 \\
\hline $15 \mathrm{H}-2$ & 27.4 & 0.30 & 44 & 1.6 & 26 & 110 & 1.7 & 11.6 & 4 & 4.0 & $<1$ & 0.6 & 10.0 & 11.0 & $<3$ & 1.9 & 90 \\
\hline $15 \mathrm{H}-3$ & 29.2 & 0.32 & 31 & 1.7 & 23 & 100 & 2.5 & 12.1 & $<3$ & 4.0 & $<1$ & 0.6 & 10.0 & 8.7 & $<3$ & 2.1 & 90 \\
\hline $15 \mathrm{H}-3$ & 33.5 & 0.36 & $<5$ & 1.7 & 26 & 130 & 2.0 & 13.6 & $<3$ & 4.5 & 1 & 0.7 & 12.0 & 4.3 & $<3$ & 2.2 & 80 \\
\hline $15 \mathrm{H}-3$ & 32.2 & 0.35 & 5 & 1.5 & 23 & 120 & 1.8 & 13.6 & $<3$ & 4.5 & 1 & 0.7 & 11.0 & 4.7 & $<3$ & 2.3 & 110 \\
\hline $15 \mathrm{H}-3$ & 31.0 & 0.31 & 7 & 1.6 & 23 & 120 & 1.7 & 13.6 & $<3$ & 4.2 & $<1$ & 0.6 & 12.0 & 4.6 & 3 & 2.0 & 90 \\
\hline $15 \mathrm{H}-3$ & 28.5 & 0.30 & 8 & 1.6 & 21 & 100 & 1.4 & 12.2 & $<3$ & 4.0 & 1 & 0.5 & 10.0 & 5.7 & $<3$ & 2.0 & 90 \\
\hline $15 \mathrm{H}-4$ & 30.1 & 0.31 & 12 & 1.6 & 21 & 110 & 1.2 & 12.2 & $<3$ & 4.1 & $<1$ & 0.5 & 10.0 & 9.4 & $<3$ & 2.0 & 90 \\
\hline $15 \mathrm{H}-4$ & 32.7 & 0.33 & 10 & 1.6 & 25 & 130 & 1.8 & 13.1 & $<3$ & 4.4 & 1 & 0.7 & 12.0 & 5.6 & $<3$ & 2.2 & 110 \\
\hline $15 \mathrm{H}-4$ & 31.2 & 0.33 & 8 & 1.5 & 24 & 110 & 1.9 & 12.5 & 4 & 4.3 & 1 & 0.6 & 11.0 & 3.7 & 4 & 2.2 & 130 \\
\hline $15 \mathrm{H}-4$ & 29.2 & 0.31 & 9 & 1.6 & 24 & 110 & 1.3 & 12.0 & $<3$ & 4.0 & 1 & 0.6 & 11.0 & 4.7 & $<3$ & 2.0 & 110 \\
\hline $15 \mathrm{H}-4$ & 27.2 & 0.30 & 9 & 1.7 & 20 & 90 & 1.3 & 11.3 & $<3$ & 3.7 & $<1$ & 0.7 & 10.0 & 4.9 & $<3$ & 1.9 & 80 \\
\hline $15 \mathrm{H}-5$ & 26.5 & 0.26 & 6 & 1.7 & 15 & 90 & 1.2 & 11.0 & $<3$ & 3.6 & $<1$ & 0.6 & 9.2 & 5.1 & 3 & 1.7 & 90 \\
\hline $15 \mathrm{H}-5$ & 25.5 & 0.26 & 10 & 1.7 & 17 & 100 & 1.4 & 11.0 & $<3$ & 3.5 & $<1$ & 0.5 & 9.2 & 3.9 & $<3$ & 1.7 & 90 \\
\hline $15 \mathrm{H}-5$ & 23.4 & 0.25 & 6 & 1.6 & 20 & 60 & 1.1 & 10.0 & $<3$ & 3.2 & 1 & $<0.5$ & 8.4 & 4.0 & $<3$ & 1.6 & 90 \\
\hline $15 \mathrm{H}-5$ & 23.9 & 0.23 & 6 & 1.7 & 18 & 80 & 1.1 & 10.1 & $<3$ & 3.3 & $<1$ & 0.5 & 8.7 & 4.8 & $<3$ & 1.5 & 100 \\
\hline $15 \mathrm{H}-6$ & 25.9 & 0.26 & 9 & 1.6 & 22 & 90 & 1.1 & 10.8 & $<3$ & 3.4 & $<1$ & 0.5 & 9.2 & 4.7 & 3 & 1.7 & 110 \\
\hline $15 \mathrm{H}-6$ & 27.6 & 0.27 & 10 & 1.7 & 21 & 120 & 1.1 & 11.6 & $<3$ & 3.8 & $<1$ & 0.6 & 10.0 & 4.7 & $<3$ & 1.8 & 110 \\
\hline $15 \mathrm{H}-6$ & 28.1 & 0.27 & 9 & 1.6 & 25 & 100 & 1.5 & 12.0 & $<3$ & 3.9 & $<1$ & 0.5 & 10.0 & 4.4 & $<3$ & 1.9 & 90 \\
\hline $15 \mathrm{H}-6$ & 31.1 & 0.33 & 19 & 1.8 & 25 & 130 & 1.5 & 13.5 & 3 & 5.0 & $<1$ & 0.7 & 12.0 & 8.3 & $<3$ & 2.2 & 130 \\
\hline $15 \mathrm{H}-6$ & 27.9 & 0.29 & 62 & 1.7 & 24 & 120 & 1.6 & 11.3 & 7 & 4.1 & $<1$ & 0.5 & 9.7 & 14.5 & $<3$ & 2.0 & 110 \\
\hline $15 \mathrm{H}-7$ & 24.3 & 0.26 & 58 & 1.8 & 20 & 80 & 1.5 & 10.4 & 4 & 3.5 & $<1$ & 0.6 & 8.1 & 12.4 & $<3$ & 1.8 & 100 \\
\hline $15 \mathrm{H}-7$ & 29.6 & 0.39 & 35 & 1.8 & 23 & 110 & 2.2 & 11.6 & $<3$ & 3.9 & 1 & 0.6 & 9.9 & 7.3 & $<3$ & 2.2 & 90 \\
\hline $15 \mathrm{H}-7$ & 35.7 & 0.33 & 7 & 1.6 & 25 & 140 & 2.7 & 14.7 & $<3$ & 4.8 & 1 & 0.6 & 13.0 & 4.7 & $<3$ & 2.2 & 110 \\
\hline $15 \mathrm{H}-7$ & 32.4 & 0.32 & 7 & 1.7 & 20 & 130 & 1.7 & 14.3 & $<3$ & 4.5 & 1 & 0.6 & 13.0 & 6.5 & 3 & 2.0 & 110 \\
\hline $15 \mathrm{H}-8$ & 32.4 & 0.32 & 13 & 1.6 & 23 & 140 & 1.7 & 13.6 & $<3$ & 4.4 & 1 & 0.6 & 12.0 & 5.2 & 3 & 2.1 & 110 \\
\hline $15 \mathrm{H}-8$ & 30.1 & 0.33 & 10 & 1.5 & 23 & 110 & 1.9 & 12.7 & $<3$ & 4.2 & 1 & 0.7 & 11.0 & 5.7 & $<3$ & 2.2 & 100 \\
\hline $15 \mathrm{H}-8$ & 28.6 & 0.31 & $<5$ & 1.7 & 24 & 100 & 1.4 & 12.0 & $<3$ & 3.9 & 1 & 0.7 & 10.0 & 4.4 & $<3$ & 1.9 & 100 \\
\hline $15 \mathrm{H}-\mathrm{CC}$ & 30.8 & 0.33 & 14 & 1.7 & 21 & 110 & 1.4 & 12.4 & $<3$ & 4.2 & 1 & 0.5 & 11.0 & 5.0 & $<3$ & 2.1 & 110 \\
\hline
\end{tabular}

Depths, which indicate the top of the sampled interval, have been taken from Bristow and deMenocal (this volume). 
Table 2. Detection limits for INAA method.

\begin{tabular}{|c|c|c|c|}
\hline Element & Symbol & $\begin{array}{l}\text { Detection } \\
\text { limit } \\
\text { (ppm) }\end{array}$ & $\begin{array}{c}\text { Standard } \\
\text { deviation } \\
(\%)\end{array}$ \\
\hline Silver & $\mathrm{Ag}$ & 5 & \\
\hline Arsenic & As & 2 & 7.1 \\
\hline Gold & $\mathrm{Au}$ & $5 \mathrm{ppb}$ & \\
\hline Barium & $\mathrm{Ba}$ & 100 & \\
\hline Bromine & $\mathrm{Br}$ & 1 & 2.6 \\
\hline Calcium & $\mathrm{Ca}$ & $0.5 \%$ & \\
\hline Cerium & $\mathrm{Ce}$ & 3 & 9.1 \\
\hline Cobalt & $\mathrm{Co}$ & 1 & 0.4 \\
\hline Chromium & $\mathrm{Cr}$ & 2 & \\
\hline Cesium & Cs & 1 & 0.0 \\
\hline Europium & Eu & 0.2 & 6.1 \\
\hline Iron & $\mathrm{Fe}$ & $0.05 \%$ & \\
\hline Hafnium & $\mathrm{Hf}$ & 0.5 & 4.4 \\
\hline Lanthanum & $\mathrm{La}$ & 0.5 & 1.5 \\
\hline Lutetium & $\mathrm{Lu}$ & 0.05 & 2.9 \\
\hline Molybdenum & Mo & 5 & 12.9 \\
\hline Sodium & $\mathrm{Na}$ & 100 & \\
\hline Neodymium & $\mathrm{Nd}$ & 5 & 3.4 \\
\hline Rubidium & $\mathrm{Rb}$ & 20 & \\
\hline Antimony & $\mathrm{Sb}$ & 0.2 & 8.8 \\
\hline Scandium & Sc & 0.1 & 1.8 \\
\hline Selenium & $\mathrm{Se}$ & 3 & \\
\hline Samarium & $\mathrm{Sm}$ & 0.1 & \\
\hline Tantalum & $\mathrm{Ta}$ & 1 & \\
\hline Terbium & $\mathrm{Tb}$ & 0.5 & 3.8 \\
\hline Thorium & Th & 0.5 & 4.8 \\
\hline Uranium & $\mathrm{U}$ & 0.5 & 1.0 \\
\hline Tungsten & W & 3 & \\
\hline Ytterbium & $\mathrm{Yb}$ & 0.2 & 1.4 \\
\hline Zinc & $\mathrm{Zn}$ & 40 & \\
\hline
\end{tabular}

